\title{
Existence and Multiplicity of Nonnegative Solutions for Quasilinear Elliptic Exterior Problems with Nonlinear Boundary Conditions
}

\author{
Jincheng Huang ${ }^{1,2}$ \\ ${ }^{1}$ Department of Mathematics and Physics, Hohai University, Changzhou Campus, Changzhou 213022, China \\ ${ }^{2}$ College of Sciences, Hohai University, Nanjing 210098, China \\ Correspondence should be addressed to Jincheng Huang; slbhuangjc@163.com
}

Received 21 May 2013; Revised 27 October 2013; Accepted 27 October 2013

Academic Editor: Julio Rossi

Copyright (c) 2013 Jincheng Huang. This is an open access article distributed under the Creative Commons Attribution License, which permits unrestricted use, distribution, and reproduction in any medium, provided the original work is properly cited.

Existence and multiplicity results are established for quasilinear elliptic problems with nonlinear boundary conditions in an exterior domain. The proofs combine variational methods with a fibering map, due to the competition between the different growths of the nonlinearity and nonlinear boundary term.

\section{Introduction}

Consider the following quasilinear elliptic problem:

$$
\begin{gathered}
-\operatorname{div}\left(a(x)|\nabla u|^{p-2} \nabla u\right)+b(x)|u|^{p-2} u=h(x, u), \quad \text { in } \Omega, \\
a(x)|\nabla u|^{p-2} \frac{\partial u}{\partial n}+g(x)|u|^{p-2} u=0, \quad \text { on } \Gamma=\partial \Omega,
\end{gathered}
$$

where $\Omega$ is a smooth exterior domain in $\mathbb{R}^{N}, 1<p<N$, and $n$ is the unit vector of the outward normal on the boundary $\Gamma=\partial \Omega$.

Equations of the type (1) arise in many and diverse contexts like differential geometry [1], nonlinear elasticity [2], non-Newtonian fluid mechanics [3], glaciology [4], and mathematical biology [5]. As a result, questions concerning the solvability of problem (1) have received great attention; see [6-10].

For $h(x, u)=c(x)|u|^{q-2} u-d(x)|u|^{s-2} u-e(x)|u|^{t-2} u$ with $q, s \in\left(1, p^{*}\right), t>p^{*}$, by using the fibering method, Kandilakis and Lyberopoulos [6] studied the existence of nonnegative solutions for problem (1) in unbounded domains with a noncompact boundary. When $h(x, u)=c(x)|u|^{q-2} u-$ $d(x)|u|^{s-2} u$ with $q, s \in\left(1, p^{*}\right)$, Lyberopoulos [7] studied the existence versus absence of nontrivial weak solutions for problem (1). Similar consideration can be found in Kandilakis and Magiropoulos [8]. In [9], Filippucci et al. established existence and nonexistence results for problem (1) via variational methods combined with the geometrical feature, where $h(x, u)=\lambda g(x)|u|^{r-2} u-|u|^{q-2} u$. Recently, Chen et al. [10] considered the existence and multiple of solutions for problem (1) by the variational principle and the mountain pass lemma.

Motivated by these findings, we consider the following quasilinear elliptic problem:

$$
\begin{array}{r}
-\operatorname{div}\left(a(x)|\nabla u|^{p-2} \nabla u\right)+b(x)|u|^{p-2} u=f(x)|u|^{q-2} u, \\
\text { in } \Omega, \\
a(x)|\nabla u|^{p-2} \frac{\partial u}{\partial n}+g(x)|u|^{r-2} u=0, \quad \text { on } \Gamma=\partial \Omega,
\end{array}
$$

where $\Omega$ is a smooth exterior domain in $\mathbb{R}^{N}$ and $n$ is the unit vector of the outward normal on the boundary $\Gamma=\partial \Omega$. Since $r \neq p$, problem (2) is essentially different from problem (1). Using the Nehari manifold and fibering map, $\mathrm{Wu}$ [11] considered problem (2) for $p=2$; Afrouzi and Rasouli [12] considered problem (2) for $1<p<N$. 
Throughout this paper, we make the following assumptions.

$\left(H_{1}\right) 1<p<N, 1<q<p^{*}$, and $1<r<p_{*}$, where $p^{*}=N p /(N-p)$ and $p_{*}=(N-1) p /(N-p)$.

$\left(H_{2}\right)$ The function $a(x) \geq a_{0}>0$ and $a(x) \in L^{\infty}(\Omega)$.

$\left(H_{3}\right)$ The function $b(x) \geq b_{0}>0$ and $b(x) \in L^{\infty}(\Omega)$.

$\left(H_{4}\right)$ The function $f(x)$ satisfies $\Omega_{f}^{+}:=\{x \in \Omega: f(x)>$ $0\} \neq \emptyset$ and $f(x) \in L^{\delta}(\Omega)$ with $p /(p-q) \geq \delta>q_{0}=$ $p^{*} /\left(p^{*}-q\right)$.

$\left(H_{5}\right)$ The function $g(x) \geq 0, g(x) \not \equiv 0$ in $\Gamma$ and $g(x) \epsilon$ $L^{\sigma}(\Gamma)$ with $p /(p-r) \geq \sigma>r_{0}=p_{*} /\left(p_{*}-r\right)$.

The purpose of this paper is to find existence and multiplicity of nonnegative solutions to problem (2). Our proofs are based on the variational method. The main difficulty is the lack of compactness of the Sobolev embeddings in unbounded domains. To overcome this difficulty, we impose the integrality conditions $\left(H_{4}\right)-\left(H_{5}\right)$ on $f$ and $g$ to establish compact Sobolev embedding theorems (see Lemmas 3 and 4).

The rest of the paper is organized as follows. In Section 2, we set up the variational framework of the problem and give some preliminaries. Section 3 is devoted to the existence results for problem (2). The multiplicity of nonnegative solutions for problem (2) is considered in the last section.

\section{Variational Framework and Some Preliminaries}

In this section, we set up the variational framework and give some preliminaries.

Define the weighted Sobolev space $E$ as the completion of $C_{0}^{\infty}\left(\mathbb{R}^{N}\right)$ under the norm

$$
\|u\|_{E}^{p}=\int_{\Omega}\left(|a(x) \nabla u|^{p}+b(x)|u|^{p}\right) d x
$$

which is equivalent to the standard one under assumptions $\left(H_{2}\right)-\left(H_{3}\right)$. Moreover, denote by $L^{q}(\Omega ;|f|)$ and $L^{r}(\Gamma ; g)$ the weighted Lebesgue spaces equipped with the norm:

$$
\begin{aligned}
\|u\|_{L^{q}(\Omega ;|f|)}^{q} & =\int_{\Omega}|f(x)||u|^{q} d x, \\
\|u\|_{L^{r}(\Gamma ; g)}^{r} & =\int_{\Gamma} g(x)|u|^{r} d S,
\end{aligned}
$$

respectively. The definition of the weak solution of problem (2) reads as follows.

Definition 1. One says $u \in E$ is a weak solution of problem (2) if

$$
\begin{aligned}
\int_{\Omega} a(x)|\nabla u|^{p-2} \nabla u \nabla v d x+\int_{\Omega} b(x)|u|^{p-2} u v d x \\
\quad+\int_{\Gamma} g(x)|u|^{r-2} u v d S=\int_{\Omega} f(x)|u|^{q-2} u v d x
\end{aligned}
$$

holds for all $v \in E$.
The energy functional corresponding to problem (2) is

$$
J(u)=\frac{1}{p}\|u\|_{E}^{p}+\frac{1}{r} G(u)-\frac{1}{q} F(u),
$$

where $G(u)=\int_{\Gamma} g(x)|u|^{r} d S, F(u)=\int_{\Omega} f(x)|u|^{q} d x$. It is well known that the weak solutions of (2) are the critical points of the energy functional $J(\cdot)$. If $u$ is a critical point of $J(\cdot)$, then necessarily $u$ belongs to the Nehari manifold:

$$
\mathcal{N}=\left\{u \in E \backslash\{0\}:\left\langle J^{\prime}(u), u\right\rangle=0\right\},
$$

where

$$
\left\langle J^{\prime}(u), u\right\rangle=\|u\|_{E}^{p}+G(u)-F(u) .
$$

For all $u \in \mathcal{N}$, we have $u \in F_{+}$, where

$$
F_{+}=\{F(u)>0\} .
$$

Moreover

$$
\begin{aligned}
J(u) & =\left(\frac{1}{p}-\frac{1}{q}\right)\|u\|_{E}^{p}+\left(\frac{1}{r}-\frac{1}{q}\right) G(u) \\
& =\left(\frac{1}{p}-\frac{1}{r}\right)\|u\|_{E}^{p}+\left(\frac{1}{r}-\frac{1}{q}\right) F(u) \\
& =\left(\frac{1}{p}-\frac{1}{q}\right) F(u)+\left(\frac{1}{r}-\frac{1}{p}\right) G(u) .
\end{aligned}
$$

The variational framework that we adopt is based on the so-called one-dimensional fibering method proposed by Pohozaev [13]. The central idea of this strategy consists in embedding the original variational problem into the "wider" space $E_{1}:=\mathbb{R} \times E$ and then investigating the conditional solvability of the new problem in $E_{1}$ under an appropriately imposed constraint. To this end, we define the extended functional $\phi:[0, \infty) \times E \rightarrow \mathbb{R}$ by setting for any $v \in E$

$$
\phi(t, v)=J(t v)=\frac{t^{p}}{p}\|v\|_{E}^{p}+\frac{t^{r}}{r} G(v)-\frac{t^{q}}{q} F(v), \quad t \geq 0 .
$$

If $u=t v$ is a critical point of $J(\cdot)$, then necessarily $\phi_{t}(t, v)=0$; that is,

$$
t^{p-1}\|v\|_{E}^{p}+t^{r-1} G(v)-t^{q-1} F(v)=0 .
$$

In particular, if $t \neq 0$, then (12) is equivalent to

$$
\psi(t, v)=\|v\|_{E}^{p},
$$

where

$$
\psi(t, v)=t^{q-p} F(v)-t^{r-p} G(v) .
$$

Now, suppose that $t=t(v) \geq 0$ solves (13) for all $v \in E \backslash\{0\}$; then $t \in C^{1}(E)$. Furthermore, if $t(v)>0$ exists and is unique for all $v \in E \backslash\{0\}$, then (13) generates a bijection between $E \backslash\{0\}$ and $\mathscr{N}$. Moreover, the following proposition holds. 
Lemma 2 (see [13]). If $v$ is a conditional critical point of $\Phi(\cdot)$, under the constraint $\|v\|_{E}=1$, then $u=t(v) v$ is a critical point of $J(\cdot)$, where $\Phi(v)=J(t(v) v)$ and $t(v)$ is a nonnegative solution of (13).

In view of Lemma 2, the problem of finding solutions of (2) will be reduced to that of locating the critical point of $\Phi(\cdot)$ under the constraint $\|v\|_{E}=1$.

The following compact embedding theorems play an important role in the proof of our main results.

Lemma 3. Assume $\left(\mathrm{H}_{2}\right)-\left(\mathrm{H}_{4}\right)$. Then the embedding $E \hookrightarrow$ $L^{q}(\Omega ;|f|)$ is compact.

Proof. Let $u \in E$. Since $p /(p-q) \geq \delta>q_{0}$, it follows that $p \leq q \delta^{\prime}=q \delta /(\delta-1)<p^{*}$. Let $W^{1, p}(\Omega)$ be the standard Banach space endowed with the norm $\|u\|_{W^{1, p}(\Omega)}^{p}=\int_{\Omega}\left(|\nabla u|^{p_{+}}\right.$ $\left.|u|^{p}\right) d x$. By assumptions $\left(H_{2}\right)-\left(H_{3}\right), E \sim W^{1, p}(\Omega)$. Similar to the proof of [10, Lemma 2] (see also the proof of [14, Theorem $7.9])$, we can prove that $W^{1, p}(\Omega) \hookrightarrow L^{q \delta^{\prime}}(\Omega)$ is compact and so is $E \hookrightarrow L^{q \delta^{\prime}}(\Omega)$. Let $S_{1}$ be the best trace embedding constant; that is,

$$
S_{1}=\inf _{u \in E, u \neq 0} \frac{\|u\|_{E}^{p}}{\|u\|_{L^{q \delta^{\prime}}(\Omega)}^{p}} .
$$

By Hölder's inequality, we have

$$
\begin{aligned}
\int_{\Omega}|f(x)||u|^{q} d x & \leq\left(\int_{\Omega}|f|^{\delta} d x\right)^{1 / \delta}\left(\int_{\Omega}|u|^{q \delta^{\prime}} d x\right)^{1 / \delta^{\prime}} \\
& \leq S_{1}^{-q / p}\||f|\|_{L^{\delta}(\Omega)}\|u\|_{E}^{q} .
\end{aligned}
$$

This shows that the embedding $E \hookrightarrow L^{q}(\Omega ;|f|)$ is continuous.

Assume $\left\{u_{n}\right\}$ is a bounded sequence in $E$. Then by the compact embedding $E \hookrightarrow L^{q \delta^{\prime}}(\Omega)$, there exist $u \in E$ and a subsequence of $\left\{u_{n}\right\}$ (not relabelled) such that $u_{n} \rightarrow u$ strongly in $L^{q \delta^{\prime}}(\Omega)$.

By Hölder's inequality again, we infer

$$
\begin{aligned}
& \int_{\Omega}|f(x)|\left|u_{n}-u\right|^{q} d x \\
& \leq\left(\int_{\Omega}|f|^{\delta} d x\right)^{1 / \delta}\left(\int_{\Omega}\left|u_{n}-u\right|^{q \delta^{\prime}} d x\right)^{1 / \delta^{\prime}} \longrightarrow 0 \\
& \text { as } n \longrightarrow \infty .
\end{aligned}
$$

This completes the proof.

Lemma 4. Assume $\left(\mathrm{H}_{2}\right)-\left(\mathrm{H}_{3}\right)$ and $\left(\mathrm{H}_{5}\right)$. Then the embedding $E \hookrightarrow L^{r}(\Gamma ; g)$ is compact.

Proof. Let $u \in E$. Since $p /(p-r) \geq \sigma>r_{0}$, it follows that $p \leq$ $r \sigma^{\prime}=r \sigma /(\sigma-1)<p_{*}$. Hence the embedding $E \hookrightarrow L^{r \sigma^{\prime}}(\Gamma)$ is compact (see $[15,16])$. Let $S_{2}$ be the best trace embedding constant; that is,

$$
S_{2}=\inf _{u \in E, u \neq 0} \frac{\|u\|_{E}^{p}}{\|u\|_{L^{r^{\prime}}(\Gamma)}^{p}} .
$$

By Hölder's inequality, we have

$$
\begin{aligned}
\int_{\Gamma} g(x)|u|^{r} d S & \leq\left(\int_{\Gamma} g^{\sigma} d S\right)^{1 / \sigma}\left(\int_{\Gamma}|u|^{r \sigma^{\prime}} d S\right)^{1 / \sigma^{\prime}} \\
& \leq S_{2}^{-r / p}\|g\|_{L^{\sigma}(\Gamma)}\|u\|_{E}^{r} .
\end{aligned}
$$

This shows that the embedding $E \hookrightarrow L^{r}(\Gamma ; g)$ is continuous.

Assume $\left\{u_{n}\right\}$ is a bounded sequence in $E$. Then by the compact embedding $E \hookrightarrow L^{r \sigma^{\prime}}(\Gamma)$, there exist $u \in E$ and a subsequence of $\left\{u_{n}\right\}$ (not relabelled) such that $u_{n} \rightarrow u$ strongly in $L^{r \sigma^{\prime}}(\Gamma)$.

By Hölder's inequality again, we infer

$$
\begin{aligned}
& \int_{\Gamma} g(x)\left|u_{n}-u\right|^{r} d S \\
& \quad \leq\left(\int_{\Gamma} g^{\sigma} d S\right)^{1 / \sigma}\left(\int_{\Gamma}\left|u_{n}-u\right|^{r \sigma^{\prime}} d S\right)^{1 / \sigma^{\prime}} \longrightarrow 0 \quad \text { as } n \longrightarrow \infty
\end{aligned}
$$

This completes the proof. 18]).

We also need the following mountain pass lemma (see [17,

Lemma 5. Let $E$ be a real Banach space and $J \in C^{1}(E, \mathbb{R})$ with $J(0)=0$. Suppose

$\left(A_{1}\right)$ there are $\rho, \alpha>0$ such that $J(u) \geq \alpha$ for $\|u\|_{E}=\rho$; $\left(A_{2}\right)$ there is $e \in E,\|e\|_{E}>\rho$ such that $J(e)<0$.

Define

$$
\Gamma_{0}=\left\{\gamma \in C^{1}([0,1], E) \mid \gamma(0)=0, \gamma(1)=e\right\} .
$$

Then

$$
c=\inf _{\gamma \in \Gamma_{0} 0 \leq t \leq 1} J(\gamma(t)) \geq \alpha
$$

is finite and $J(\cdot)$ possess a $(P S)_{c}$ sequence at level c. Furthermore, if $J$ satisfies the (PS) condition, then $c$ is a critical value of $J$.

To get multiplicity results, we need the following fountain theorem due to Bartsch [19] and a critical point theorem in $[20,21]$

Let $X$ be a reflexive and separable Banach space. It is well known that there exist $e_{j} \in X$ and $e_{j}^{*} \in X^{*}(j=1,2, \ldots)$ such that

(1) $\left\langle e_{i}, e_{j}^{*}\right\rangle=\delta_{i j}$, where $\delta_{i j}=1$ for $i=j$ and $\delta_{i j}=0$ for $i \neq j$, 
(2) $X=\overline{\operatorname{span}\left\{e_{1}, e_{2}, \ldots\right\}}$ and $X^{*}=\overline{\operatorname{span}\left\{e_{1}^{*}, e_{2}^{*}, \ldots\right\}}$.

For convenience, we write

$$
\begin{array}{r}
X_{j}=\operatorname{span}\left\{e_{j}\right\}, \quad Y_{k}=\bigoplus_{j=1}^{k} X_{j}, \quad Z_{k}=\overline{\bigoplus_{j=k}^{\infty} X_{j},} \\
j, k=1,2, \ldots .
\end{array}
$$

Lemma 6 (fountain theorem [19]). Assume $J \in C^{1}\left(X, \mathbb{R}^{1}\right)$ is an even functional that satisfies the $(P S)_{c}$ condition. If for every $k \in \mathbb{N}$ there exist $\rho_{k}>r_{k}>0$ such that

$$
\begin{aligned}
& \left(B_{1}\right) a_{k}:=\inf _{u \in Z_{k},\|u\|_{X}=r_{k}} J(u) \rightarrow \infty \text { as } k \rightarrow \infty, \\
& \left(B_{2}\right) b_{k}:=\max _{u \in Y_{k},\|u\|_{X}=\rho_{k}} J(u) \leq 0,
\end{aligned}
$$

then $J$ has a sequence of critical points $\left\{u_{k}\right\}$ with $J\left(u_{k}\right) \rightarrow \infty$.

Lemma 7 (see $[20,21])$. Let $J \in C^{1}\left(X, \mathbb{R}^{1}\right)$, where $X$ is a Banach space. Assume that $J$ satisfies the $(P S)_{c}$ condition and is even and bounded from below, and $J(0)=0$. If for any $k \in \mathbb{N}$ there exists a $k$-dimensional subspace $Y_{k}$ and $\rho_{k}>0$ such that

$$
\sup _{u \in Y_{k},\|u\|_{X}=\rho_{k}} J(u)<0,
$$

then J has a sequence of critical values $c_{k}<0$ satisfying $c_{k} \rightarrow 0$ as $k \rightarrow \infty$.

\section{Existence of Nonnegative Solutions}

In this section, the existence results are established for problem (2). The proofs combine variational methods with a fibering map. Since $J(u)=J(|u|)$, we may suppose that the solution to problem (2) is nonnegative throughout this paper.

Theorem 8. Let $\left(H_{1}\right)-\left(H_{5}\right)$ hold with either $q<\min \{p, r\}$ or $q>\max \{p, r\}$. Then problem (2) admits a nonnegative nontrivial weak solution $u \in E \backslash\{0\}$ which is also a ground state.

Proof. Suppose $q<\min \{p, r\}$. Rewriting (13) as

$$
t^{p-q}\|v\|_{E}^{p}+t^{r-q} G(v)=F(v)
$$

we immediately see that for every $v \in F_{+}$(where $F_{+}$is defined by (9)) there exists a unique $t(v)>0$ satisfying (25). Moreover, it can be easily checked that

$$
\begin{gathered}
\mu t(\mu v)=t(v), \quad \text { for } \mu>0, v \in F_{+}, \\
\Phi(v)=\left(\frac{1}{p}-\frac{1}{q}\right)(t(v))^{p}\|v\|_{E}^{p}+\left(\frac{1}{r}-\frac{1}{q}\right)(t(v))^{r} G(v)<0 \\
\forall v \in F_{+} .
\end{gathered}
$$

Consider now the variational problem

$$
M:=\inf _{v \in F_{+},\|v\|_{E}=1} \Phi(v)<0 .
$$

Let $\left\{v_{n}\right\}$ be a minimizing sequence in $F_{+}$with $\left\|v_{n}\right\|_{E}=1$. Then there exists $v_{0} \in E$ such that $v_{n} \rightarrow v_{0}$ in $E$. By Lemmas 3 and 4 , we have $F\left(v_{n}\right) \rightarrow F\left(v_{0}\right) \geq 0$ and $G\left(v_{n}\right) \rightarrow G\left(v_{0}\right) \geq 0$.
We first assert that $v_{0} \in F_{+}$. Suppose the contrary; then $F\left(v_{0}\right)=0$. In view of $(25)$,

$$
F\left(v_{n}\right) \geq\left(t\left(v_{n}\right)\right)^{p-q} .
$$

Letting $n \rightarrow \infty$, it follows that $t\left(v_{n}\right) \rightarrow 0$. Thus

$$
\begin{aligned}
\Phi\left(v_{n}\right)= & \left(\frac{1}{p}-\frac{1}{q}\right)\left(t\left(v_{n}\right)\right)^{p}\left\|v_{n}\right\|_{E}^{p} \\
& +\left(\frac{1}{r}-\frac{1}{q}\right)\left(t\left(v_{n}\right)\right)^{r} G\left(v_{n}\right) \longrightarrow 0, \quad \text { as } n \longrightarrow \infty
\end{aligned}
$$

which contradicts $M<0$.

Next, we prove $\left\|v_{0}\right\|_{E}=1$. If not, then $\left\|v_{0}\right\|_{E}<$ $\liminf _{n \rightarrow \infty}\left\|v_{n}\right\|_{E}=1$. So, there exists $\mu>1$ such that $\left\|\mu v_{0}\right\|_{E}=1$. From (25), we have

$$
\left(t\left(\mu v_{0}\right)\right)^{p-q}+\left(t\left(\mu v_{0}\right)\right)^{r-q} G\left(\mu v_{0}\right)=F\left(\mu v_{0}\right) .
$$

This and (26) yield

$$
\mu^{-p}\left(t\left(v_{0}\right)\right)^{p-q}+\left(t\left(v_{0}\right)\right)^{r-q} G\left(v_{0}\right)=F\left(v_{0}\right) .
$$

On the other hand, it follows from (29) that $\left\{t\left(v_{n}\right)\right\}$ is bounded and so there exists a subsequence (not relabelled) such that $t\left(v_{n}\right) \rightarrow t_{0}>0$. Thus by (25), we have

$$
t_{0}^{p-q}+t_{0}^{r-q} G\left(v_{0}\right)=F\left(v_{0}\right) \text {. }
$$

Hence $t_{0}<t\left(v_{0}\right)$. Notice that

$$
\Psi(t)=\left(\frac{1}{p}-\frac{1}{q}\right) t^{p}\left\|v_{0}\right\|_{E}^{p}+\left(\frac{1}{r}-\frac{1}{q}\right) t^{r} G\left(v_{0}\right)
$$

is strictly decreasing for all $t>0$; we have

$$
M=\liminf _{n \rightarrow \infty} \Phi\left(v_{n}\right) \geq \Psi\left(t_{0}\right)>\Psi\left(t\left(v_{0}\right)\right)=\Phi\left(v_{0}\right),
$$

which is a contradiction. So, $\left\|v_{0}\right\|_{E}=1$ and $v_{0}$ is a critical point of $\Phi(\cdot)$. By Lemma 2, $u=t\left(v_{0}\right) v_{0}$ is a nontrivial solution of problem (2). Since $t=t(v)$ is a unique solution of (25), then (25) generates a bijection between $E \backslash\{0\}$ and $\mathcal{N}$ and so the obtained solution is actually a ground state.

The case $q>\max \{p, r\}$ can be treated in a similar way.

Remark 9. Afrouzi and Rasouli [12] consider the following problem:

$$
-\operatorname{div}\left(|\nabla u|^{p-2} \nabla u\right)+b(x)|u|^{p-2} u=\lambda f(x)|u|^{q-2} u, \quad \text { in } \Omega,
$$

$$
|\nabla u|^{p-2} \frac{\partial u}{\partial n}=g(x)|u|^{r-2} u, \quad \text { on } \Gamma=\partial \Omega
$$

where $\Omega$ is a bounded domain in $\mathbb{R}^{N}$ and $1<q<p<$ $r<p_{*}=(N-1) p /(N-p)$. The functions $f$ and $g$ are continuous functions which change sign in $\bar{\Omega}$. Using the Nehari manifold and fibering map, they proved that problem 
(36) has at least two nontrivial nonnegative solutions if $|\lambda|$ is sufficiently small. In fact, by slight modification, we can prove that the result they established is still true if $\Omega$ is a smooth exterior domain or the parameters satisfy $1<r<p<q<$ $p^{*}=N p /(N-p)$. But for $g(x) \geq 0$, we can prove that problem (36) has at least one nontrivial nonnegative solution for $|\lambda|$ is sufficiently small via the method used in [12]. Notice that our result (Theorem 8 ) does not need $|\lambda|$ to be small.

Theorem 10. Let $\left(H_{1}\right)-\left(H_{5}\right)$ hold with $r<q<p$. Then problem (2) has a nonnegative nontrivial weak solution.

Proof. From Lemma 3, we have

$$
F(u) \leq S_{1}^{-q / p}\|f\|_{L^{\delta}(\Omega)}\|u\|_{E}^{q} .
$$

Thus

$$
J(u) \geq \frac{1}{p}\|u\|_{E}^{p}-\frac{1}{q} S_{1}^{-q / p}\|f\|_{L^{\delta}(\Omega)}\|u\|_{E}^{q} .
$$

So $J(u)$ is coercive by $p>q$.

By Lemmas 3 and 4, it is easy to verify that $J$ is weakly lower semicontinuous. So $J$ has a minimum point $u$ in $E$ and $u$ is a weak solution of (2).

In the following, we prove $\inf _{u \in E} J(u)<0$. Let $v \in$ $W_{0}^{1, p}(\Omega) \cap F_{+}$. Then

$$
J(t v)=\frac{1}{p} t^{p}\|v\|_{E}^{p}-\frac{1}{q} t^{q} F(v) .
$$

Thus $J(t v)<0$ for $t>0$ is sufficiently small. Notice that $W_{0}^{1, p}(\Omega) \cap F_{+} \subset E$; we obtain

$$
\inf _{v \in E} J(v) \leq \inf _{v \in W_{0}^{1, p}(\Omega) \cap F_{+}} J(v)<0 .
$$

Thus the minimum point of $J$ is nontrivial.

Theorem 11. Let $\left(H_{1}\right)-\left(H_{5}\right)$ hold with $p<q<r$. Then problem (2) has a nonnegative nontrivial weak solution in $W_{0}^{1, p}(\Omega) \cap F_{+}$.

Proof. Let $u \in W_{0}^{1, p}(\Omega) \cap F_{+}$. Then

$$
J(t u)=\frac{t^{p}}{p}\|u\|_{E}^{p}-\frac{t^{q}}{q} F(u) .
$$

Thus $J(t u) \geq \alpha>0$ for $t$ small and $J(t u)<0$ for $t$ large.

Let $\left\{u_{n}\right\} \subset W_{0}^{1, p}(\Omega) \cap F_{+}$satisfy $J\left(u_{n}\right) \rightarrow c$ in $E$ and $J^{\prime}\left(u_{n}\right) \rightarrow 0$ in $E^{*}$. Then

$$
c+1 \geq J\left(u_{n}\right)-\frac{1}{q}\left\langle J^{\prime}\left(u_{n}\right), u_{n}\right\rangle=\left(\frac{1}{p}-\frac{1}{q}\right)\left\|u_{n}\right\|_{E}^{p} .
$$

This shows that $\left\|u_{n}\right\|_{E}$ is bounded in $E$. Up to a subsequence, we obtain $u_{n} \rightarrow u$ in $E$. Thus

$$
\begin{aligned}
\int_{\Omega} a(x)|\nabla u|^{p-2} \nabla u \nabla\left(u_{n}-u\right) d x & \\
\quad+\int_{\Omega} b(x)|u|^{p-2} u\left(u_{n}-u\right) d x & \longrightarrow 0 .
\end{aligned}
$$

It follows from Lemmas 3 and 4 that

$$
u_{n} \longrightarrow u \quad \text { in } L^{q}(\Omega ;|f|), L^{r}(\Gamma ; g)
$$

Hence

$$
\begin{gathered}
\int_{\Omega} f(x)|u|^{q-2} u\left(u_{n}-u\right) d x \rightarrow 0, \\
\int_{\Gamma} g(x)\left|u_{n}\right|^{r-2} u_{n}\left(u_{n}-u\right) d S \longrightarrow 0, \\
B_{n}:=\int_{\Omega} f(x)\left(\left|u_{n}\right|^{q-2} u_{n}-|u|^{q-2} u\right)\left(u_{n}-u\right) d x \longrightarrow 0, \\
C_{n}:=\int_{\Gamma} g(x)\left(\left|u_{n}\right|^{r-2} u_{n}-|u|^{r-2} u\right)\left(u_{n}-u\right) d S \longrightarrow 0 .
\end{gathered}
$$

Then $(45)$ give that $\left\langle J^{\prime}(u), u_{n}-u\right\rangle \rightarrow 0$. Notice that $J^{\prime}\left(u_{n}\right) \rightarrow$ 0 ; we have

$$
\left\langle J^{\prime}\left(u_{n}\right)-J^{\prime}(u), u_{n}-u\right\rangle=A_{n}+B_{n}-C_{n} \longrightarrow 0,
$$

where

$$
\begin{aligned}
A_{n}= & \int_{\Omega} a(x)\left(\left|\nabla u_{n}\right|^{p-2} \nabla u_{n}-|\nabla u|^{p-2} \nabla u\right) \nabla\left(u_{n}-u\right) d x \\
& +\int_{\Omega} b(x)\left(\left|u_{n}\right|^{p-2} u_{n}-|u|^{p-2} u\right)\left(u_{n}-u\right) d x
\end{aligned}
$$

Using the standard inequality in $\mathbb{R}^{N}$ given by

$$
\begin{array}{r}
\left\langle|\xi|^{p-2} \xi-|\eta|^{p-2} \eta, \xi-\eta\right\rangle \geq C_{p}|\xi-\eta|^{p}, \quad p \geq 2, \\
\left\langle|\xi|^{p-2} \xi-|\eta|^{p-2} \eta, \xi-\eta\right\rangle \geq C_{p}|\xi-\eta|^{2}(|\xi|+|\eta|)^{p-2}, \\
1<p<2,
\end{array}
$$

we have from (46) that $u_{n} \rightarrow u$ in $E$. Thus $J(\cdot)$ satisfies (PS) condition. Then the assertion of this theorem follows from Lemma 5.

Next, we seek for a solution in $E \backslash W_{0}^{1, p}(\Omega)$ with $p<q<r$. In this case, we find it necessary to strengthen our hypothesis by assuming that the function $g(\cdot)$ is positive. That is, $\left(H_{5}\right)$ will be replaced by

$\left(H_{5}^{\prime}\right)$ the function $g(x)>0$ and $g(x) \in L^{\sigma}(\Gamma)$ with $p /(p-$ $r) \geq \sigma>r_{0}=p_{*} /\left(p_{*}-r\right)$.

Theorem 12. Let $\left(H_{1}\right)-\left(H_{4}\right)$ and $\left(H_{5}^{\prime}\right)$ hold with $p<q<r$. Suppose also

$$
\mathscr{M}:=\left\{(F(v))^{r-p}>\xi(G(v))^{q-p}\|v\|_{E}^{p(r-q)}\right\} \neq \emptyset,
$$

where $\xi=(r-p)^{r-p} /\left((q-p)^{q-p}(r-q)^{r-q}\right)(r / p)^{r-q}$. Then problem (2) has a nonnegative nontrivial weak solution in $E / W_{0}^{1, p}(\Omega)$. 
Proof. Define

$$
\mathscr{L}_{v_{0}}=\left\{v \in \mathscr{M}: G\left(\frac{v}{\|v\|_{E}}\right) \geq G\left(\frac{v_{0}}{\left\|v_{0}\right\|_{E}}\right)\right\} .
$$

Fix $v_{0}$ with $\mathscr{L}_{v_{0}} \neq \emptyset$. In view of assumption $\left(H_{5}^{\prime}\right)$, we have $G\left(v_{0}\right)>0$. For all $u \in \mathscr{L}_{v_{0}}$, there exist $t>0$ and $v \in \mathscr{L}_{v_{0}}$ with $\|v\|_{E}=1$ such that $u=t v$. Moreover, $G(v) \geq G\left(v_{0} /\left\|v_{0}\right\|_{E}\right)>0$. Thus

$$
J(u)=\frac{t^{p}}{p}\|v\|_{E}^{p}+\frac{t^{r}}{r} G(v)-\frac{t^{q}}{q} F(v) \longrightarrow \infty,
$$

as $t \rightarrow \infty$. Hence $J(\cdot)$ is coercive in $\mathscr{L}_{v_{0}}$. By Lemmas 3 and 4 , it is easy to verify that $J$ is weakly lower semicontinuous. So $J$ has a minimum point $u$ in $\mathscr{L}_{v_{0}}$ and $u$ is a weak solution of (2).

In the following, we prove that $\inf _{u \in \mathscr{L}_{v_{0}}} J(u)<0$. Notice that $\psi(t, v)=0$ as $t \rightarrow 0, \psi(t, v)=-\infty$ as $t \rightarrow+\infty$, and $\psi_{t}^{\prime}(t, v)=(q-p) t^{q-p-1} F(v)-(r-p) t^{r-p-1} G(v)$; we infer that $\psi(t, v)$ attain its maximum at $t_{M}(v)$, where

$$
t_{M}(v)=\left(\frac{q-p}{r-p} \frac{F(v)}{G(v)}\right)^{1 /(r-q)} .
$$

If $0<\|v\|_{E}^{p}<\psi\left(t_{M}(v), v\right)$, then (13) has exactly two solutions $t_{1}(v)$ and $t_{2}(v)$ with $0<t_{1}(v)<t_{M}(v)<t_{2}(v)$. Let $t(v)=t_{2}(v)$. We have from (11) and (12) that

$$
J(t(v) v)=\frac{q-p}{p q}(t(v))^{p}\|v\|_{E}^{p}-\frac{r-q}{q r}(t(v))^{r} G(v) .
$$

Let $v \in \mathscr{L}_{v_{0}}$. It follows that

$$
(t(v))^{r-p}>\left(t_{M}(v)\right)^{r-p}>\frac{r(q-p)\|v\|_{E}^{p}}{p(r-q) G(v)},
$$

which ensures $J(t(v) v)<0$. Thus $\inf _{u \in \mathscr{L}_{v_{0}}} J(u)<0$. This implies that the weak solution of (2) is nontrivial.

Remark 13. Condition (49) may be viewed as grading the "strength" of interaction induced by $F(u)$ and $G(u)$. Hence, qualitatively speaking, one may rephrase Theorem 12 as saying that if $p<q<r$, then problem (2) admits a nontrivial weak solution provided that $F(\cdot)$ "prevails" over $G(\cdot)$.

\section{Multiplicity of Nonnegative Solutions}

In this section, we establish multiplicity results for the cases $q>\max \{p, r\}$ and $q<\min \{p, r\}$ by Lemmas 6 and 7 , respectively. To this purpose, the assumption $\left(H_{4}\right)$ will be replaced by the following:

$\left(H_{4}^{\prime}\right)$ the function $f(x)$ satisfies $f(x)>0$ in $\Omega$ and $f(x) \in$ $L^{\delta}(\Omega)$ with $p /(p-q) \geq \delta>q_{0}=p^{*} /\left(p^{*}-q\right)$.

Theorem 14. Let $\left(H_{1}\right)-\left(H_{3}\right),\left(H_{4}^{\prime}\right)$, and $\left(H_{5}\right)$ hold with $q>$ $\max \{p, r\}$. Then problem (2) has a sequence of solutions $u_{k}$ in E with $J\left(u_{k}\right) \rightarrow \infty$ as $k \rightarrow \infty$.
Proof. We will prove this theorem by fountain theorem. The proof is divided into three steps.

(1) Let $Y_{k}$ and $Z_{k}$ be defined by (23) and $\beta_{k}=$ $\sup _{u \in Z_{k},\|u\|_{E}=1}\|u\|_{L^{q}(\Omega ; f)}$. Then it follows that $\beta_{k} \rightarrow 0$ (see [22]). Therefore, we have

$$
J(u) \geq \frac{1}{p}\|u\|_{E}^{p}-\frac{1}{q} F(u) \geq \frac{1}{p}\|u\|_{E}^{p}-\frac{1}{q} \beta_{k}^{q}\|u\|_{E}^{q} .
$$

Choosing $r_{k}=\left(q /\left(2 p \beta_{k}^{q}\right)\right)^{1 /(q-p)}$, we obtain that if $u \in$ $Z_{k},\|u\|_{E}=r_{k}$, then

$$
J(u) \geq \frac{1}{2 p} r_{k}^{p} \longrightarrow+\infty .
$$

Thus $\left(B_{1}\right)$ in Lemma 6 is proved.

(2)Since in the finite dimensional space $Y_{k}$ all norms are equivalent, there exist $C>0$ such that $F(u) \geq C\|u\|_{E}^{q}$ hold for all $u \in Y_{k}$. Thus by (19),

$$
\begin{array}{r}
J(u) \leq \frac{1}{p}\|u\|_{E}^{p}+\frac{1}{r} S_{2}^{-r / p}\|g\|_{L^{\sigma}(\Gamma)}\|u\|_{E}^{r}-\frac{1}{q} C\|u\|_{E}^{q}, \\
\forall u \in Y_{k} .
\end{array}
$$

Therefore $\left(B_{2}\right)$ in Lemma 6 is satisfied for every $\rho_{k}:=\|u\|_{E}>$ 0 large enough.

(3) Let $\left\{u_{n}\right\}$ be a (PS) sequence of $J$. Then we have

$$
\begin{aligned}
c+1 & \geq J\left(u_{n}\right)-\frac{1}{q}\left\langle J^{\prime}\left(u_{n}\right), u_{n}\right\rangle \\
& =\left(\frac{1}{p}-\frac{1}{q}\right)\left\|u_{n}\right\|_{E}^{p}+\left(\frac{1}{r}-\frac{1}{q}\right) G(u) \geq\left(\frac{1}{p}-\frac{1}{q}\right)\left\|u_{n}\right\|_{E}^{p} .
\end{aligned}
$$

Therefore $\left\{u_{n}\right\}$ is bounded in E. Similar to the proof of Theorem 11 , we can verify that $J(\cdot)$ satisfies (PS) condition.

Obviously, $J(\cdot)$ is an even functional and $J(0)=0$. Thus the assertion of Theorem 14 follows from Lemma 6 .

Theorem 15. Let $\left(H_{1}\right)-\left(H_{3}\right),\left(H_{4}^{\prime}\right)$, and $\left(H_{5}\right)$ hold with $q<$ $\min \{p, r\}$. Then problem (2) has a sequence of solutions $u_{k}$ in $E$ with $J\left(u_{k}\right)<0$ and $J\left(u_{k}\right) \rightarrow 0$ as $k \rightarrow \infty$.

Proof. Since

$$
\begin{aligned}
J(u) & =\frac{1}{p}\|u\|_{E}^{p}+\frac{1}{r} G(u)-\frac{1}{q} F(u) \\
& \geq \frac{1}{p}\|u\|_{E}^{p}-\frac{1}{q} S_{1}^{-q / p}\|f\|_{L^{\delta}(\Omega)}\|u\|_{E}^{q}
\end{aligned}
$$

and $q<p, J(\cdot)$ is coercive and bounded from below. As before, we can verify that (PS) condition holds.

Let $Y_{k}$ be defined by (23) and $u \in Y_{k}$. Since $Y_{k}$ is a finite dimensional space and $q<\min \{p, r\}$, we can choose $\rho_{k}>0$ small enough such that

$$
\begin{aligned}
\sup _{u \in Y_{k},\|u\|_{E}=\rho_{k}} J(u) \leq & \frac{1}{p}\|u\|_{E}^{p}+\frac{1}{r} S_{2}^{-r / p}\|g\|_{L^{\sigma}(\Gamma)}\|u\|_{E}^{r} \\
& -\frac{1}{q} C\|u\|_{E}^{q}<0 .
\end{aligned}
$$

We obtain a sequence of solutions $u_{k}$ by Lemma 7 . 


\section{Acknowledgment}

This work is supported by the Fundamental Research Funds for the Central Universities (2013B09914).

\section{References}

[1] J. L. Kazdan, Prescribing the Curvature of a Riemannian Manifold, vol. 57 of CBMS Regional Conference Series in Mathematics, American Mathematical Society, Providence, RI, USA, 1985.

[2] P. G. Ciarlet, Mathematical Elasticity. Vol. I. Three-Dimensional Elasticity, vol. 20 of Studies in Mathematics and Its Applications, North-Holland, Amsterdam, The Netherlands, 1988.

[3] J. I. Díaz, Nonlinear Partial Differential Equations and Free Boundaries. Vol. I. Elliptic Equations, vol. 106 of Research Notes in Mathematics, Pitman, Boston, Mass, USA, 1985.

[4] M.-C. Pélissier and L. Reynaud, "Étude d'un modèle mathématique d'écoulement de glacier," Comptes Rendus de l'Académie des Sciences A, vol. 279, pp. 531-534, 1974.

[5] D. G. Aronson and H. F. Weinberger, "Multidimensional nonlinear diffusion arising in population genetics," Advances in Mathematics, vol. 30, no. 1, pp. 33-76, 1978.

[6] D. A. Kandilakis and A. N. Lyberopoulos, "Indefinite quasilinear elliptic problems with subcritical and supercritical nonlinearities on unbounded domains," Journal of Differential Equations, vol. 230, no. 1, pp. 337-361, 2006.

[7] A. N. Lyberopoulos, "Existence and Liouville-type theorems for some indefinite quasilinear elliptic problems with potentials vanishing at infinity," Journal of Functional Analysis, vol. 257, no. 11, pp. 3593-3616, 2009.

[8] D. A. Kandilakis and M. Magiropoulos, "Existence results for a $p$-Laplacian problem with competing nonlinearities and nonlinear boundary conditions," Electronic Journal of Differential Equations, no. 95, pp. 1-6, 2011.

[9] R. Filippucci, P. Pucci, and V. Rădulescu, "Existence and nonexistence results for quasilinear elliptic exterior problems with nonlinear boundary conditions," Communications in Partial Differential Equations, vol. 33, no. 4-6, pp. 706-717, 2008.

[10] C. Chen, S. Liu, and H. Yao, "Existence of solutions for quasilinear elliptic exterior problem with the concave-convex nonlinearities and the nonlinear boundary conditions," Journal of Mathematical Analysis and Applications, vol. 383, no. 1, pp. 111119, 2011.

[11] T.-F. Wu, "A semilinear elliptic problem involving nonlinear boundary condition and sign-changing potential," Electronic Journal of Differential Equations, no. 131, pp. 1-15, 2006.

[12] G. A. Afrouzi and S. H. Rasouli, "A variational approach to a quasilinear elliptic problem involving the $p$-Laplacian and nonlinear boundary condition," Nonlinear Analysis: Theory, Methods \& Applications, vol. 71, no. 7-8, pp. 2447-2455, 2009.

[13] S. I. Pohozaev, "On the fibering method for the solution of nonlinear boundary value problems," Proceedings of the Steklov Institute of Mathematics, vol. 192, pp. 157-173, 1992.

[14] I. Kuzin and S. Pohozaev, Entire Solutions of Semilinear Elliptic Equations, Progress in Nonlinear Differential Equations and their Applications, 33, Birkhäuser, Basel, Switzerland, 1997.

[15] L. F. O. Faria, O. H. Miyagaki, and F. R. Pereira, "Existence results for quasilinear elliptic exterior problems involving convection term and nonlinear Robin boundary conditions," Journal of Mathematical Analysis and Applications, vol. 368, no. 2, pp. 578-586, 2010.
[16] R. A. Adams, Sobolev Spaces, Academic Press, New York, NY, USA, 1975.

[17] A. Ambrosetti and P. H. Rabinowitz, "Dual variational methods in critical point theory and applications," Journal of Functional Analysis, vol. 14, pp. 349-381, 1973.

[18] M. Struwe, Variational Methods, Applications to Nonlinear Partial Differential Equations and Hamiltonian Systems, vol. 34, Springer, New York, NY, USA, 3rd edition, 2000.

[19] T. Bartsch, "Infinitely many solutions of a symmetric Dirichlet problem," Nonlinear Analysis: Theory, Methods \& Applications, vol. 20, no. 10, pp. 1205-1216, 1993.

[20] Z.-Q. Wang, "Nonlinear boundary value problems with concave nonlinearities near the origin," Nonlinear Differential Equations and Applications, vol. 8, no. 1, pp. 15-33, 2001.

[21] H.-P. Heinz, "Free Ljusternik-Schnirelman theory and the bifurcation diagrams of certain singular nonlinear problems," Journal of Differential Equations, vol. 66, no. 2, pp. 263-300, 1987.

[22] M. Willem, Minimax Theorems, Progress in Nonlinear Differential Equations and their Applications, 24, Birkhäuser, Boston, Mass, USA, 1996. 


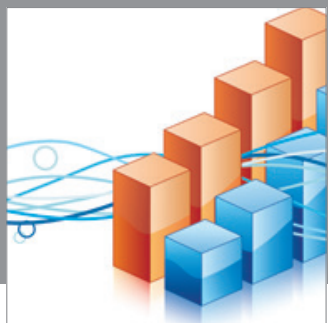

Advances in

Operations Research

mansans

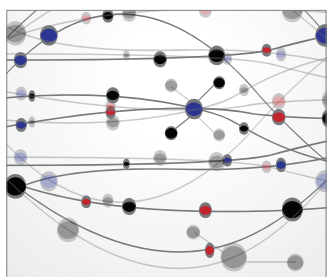

The Scientific World Journal
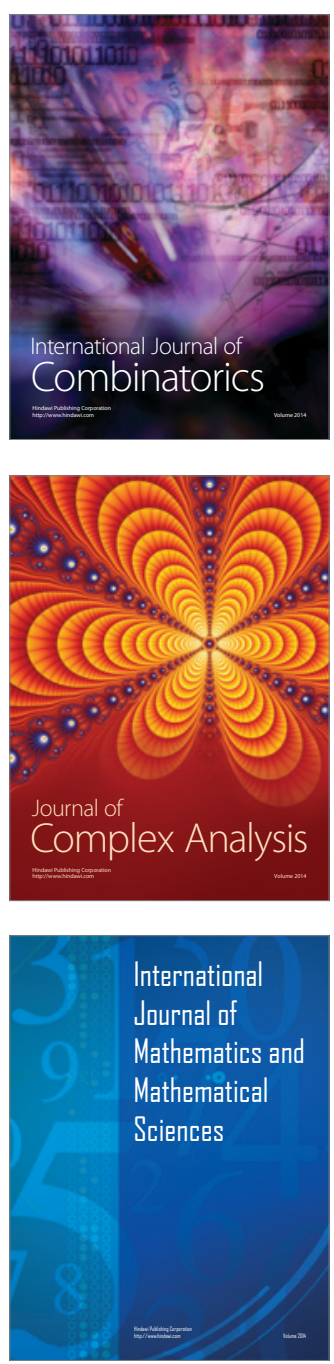
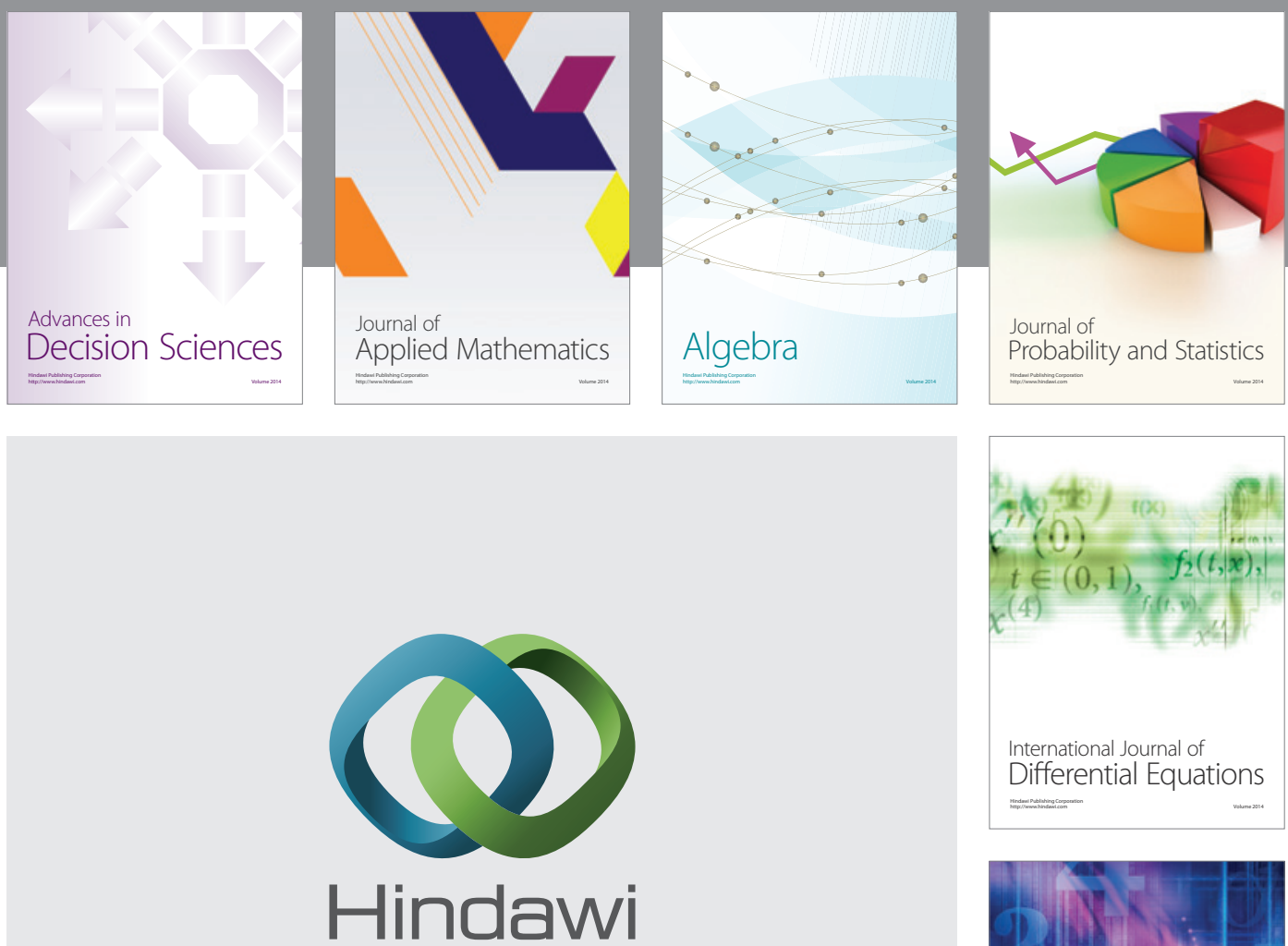

Submit your manuscripts at http://www.hindawi.com
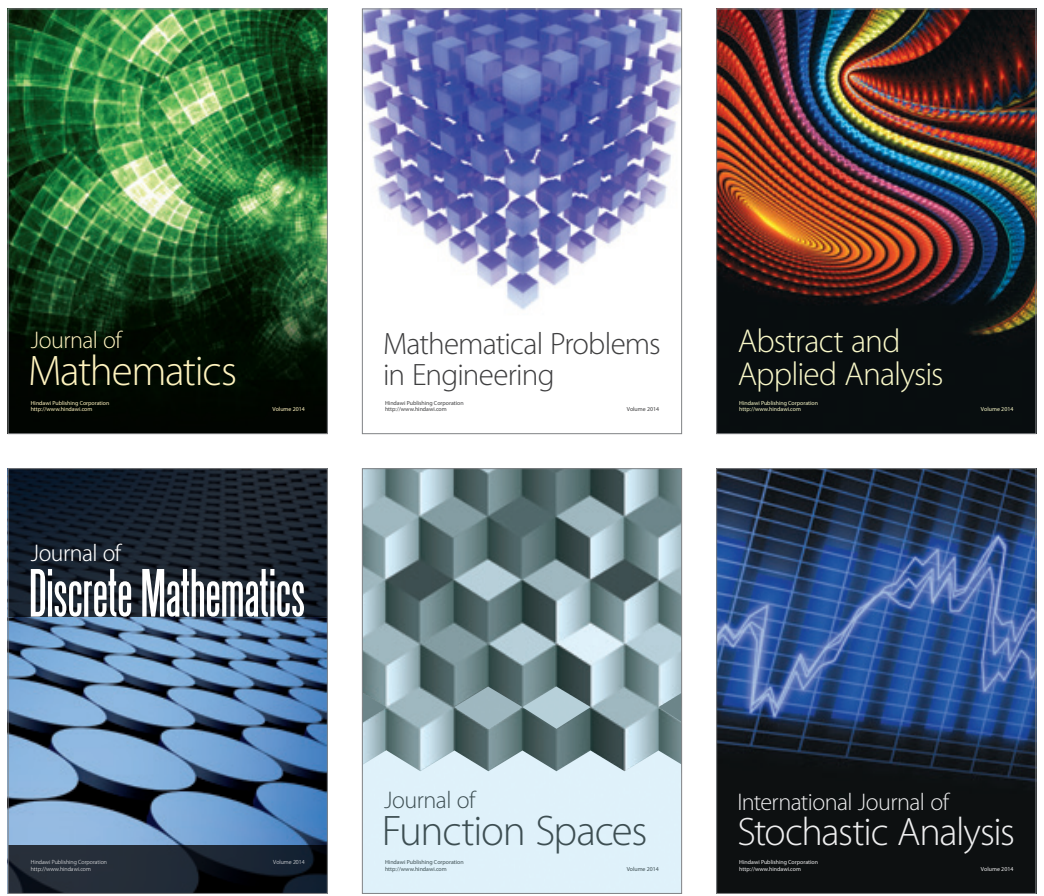

Journal of

Function Spaces

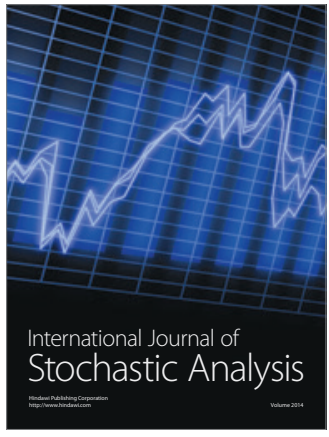

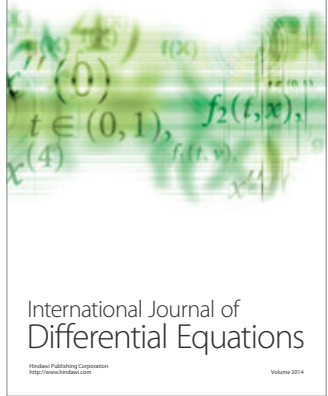
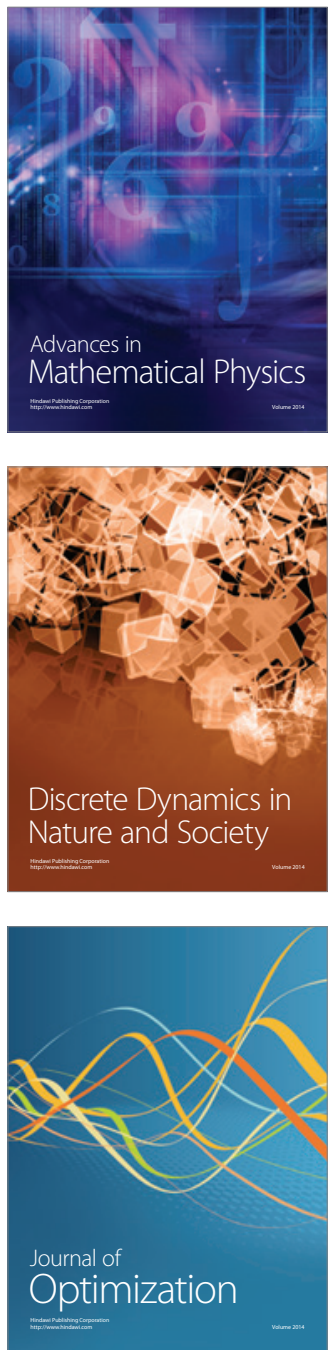